DOI: https://doi.org/10.24297/jam.v17i0.8279

\title{
Breaking Boundaries: Discovering the Impossible Counterproof of Beal's Conjecture
}

\author{
Halima Jibril Mohamed ${ }^{1}$, Adela Zyfi ${ }^{1}$, Ghedlawit Futzum ${ }^{1}$ \\ ${ }^{1}$ Discover6ix Corp,P.O. Box 11701, Etobicoke, Ontario M9C2A0 \\ discover6ix@gmail.com, adela.zyfi@yahoo.com
}

\begin{abstract}
This paper will attempt to logically differentiate between two types of fractions and discuss the idea of "zero" as a neutral integer. The paper analyzes in detail the concepts and definitions of fractions, decimals, and integers in relation to Beal's conjecture, and defines the idea of what is whole and what is a part. The arguments are then used to show proof of a counterexample for Beal's conjecture.
\end{abstract}

\section{Introduction}

For hundreds of years, mathematicians have been intrigued by the famous conjecture of Pierre Fermat, named Fermat's Last Theorem. Fermat's Last Theorem is a number theory conjecture that states: [1]

No three positive integers, $A, B, C$ can satisfy:

$$
A^{N}+B^{N}=C^{N} \text { for any integer value of } N>2
$$

Due to its lack of proof for many years, in addition to hundreds of failed attempts at further understanding the theorem, it was often believed to be untrue. [1] After hundreds of years of effort, Andrew Wiles provided the first proof for this theorem in 1994. [2] Fermat's Last Theorem has been and continues to be extremely powerful and influential in the field of mathematics, making it possible to prove a large part of the modularity theorem and allowed many other mathematical problems to be solved using new approaches. [1] It is one of the most well known mathematical theorems and has been recognized by the Guinness Book of World Records as the most complicated mathematical problem, due to the largest number of unsuccessful proofs. [3] Due to its importance and applicability, in 1993, Andrew Beal developed Beal's conjecture, a new conjecture discovered by mathematician Andrew Beal while studying generalizations of Fermat's Last Theorem. Beal's conjecture states: [4]

$A^{x}+B^{y}=C^{z}$

Where $A, B, C, x, y$, and $z$ are positive integers with $x, y, z>2$,

Then, $A, B$, and $C$ have a common prime factor

This number theory conjecture demonstrates that no solutions in positive integers $A, B, C, x, y, z$ exist, with $A, B$, and $C$ being pairwise coprime and all of $x, y, z$ being greater than 2. [4]

Literature searches show that no counterproof or counterargument of Beal's conjecture has been published so far, demonstrating the truthfulness of his statement. However, this paper aims to show and demonstrate through examples that a counterproof for the conjecture does exist.

\section{Definition of Terms}

1. Whole Number: An integer that is 0 or greater. Examples of whole numbers are $0,1,2,3,4$ to infinity. 
2. Integer: This term will be explained using two different definitions:

3. $\mathbf{1}^{\text {st }}$ definition - A number that can be written without a fractional component. Examples of this are 14, 12 , 0 , etc.

4. $\mathbf{2}^{\text {nd }}$ definition - A thing complete in itself.

5. Non-Integer - A non-integer is a number that is written with a fractional component. Examples of this are $4 \frac{1}{2}, 21 / 2,1.23$, etc.

\section{Results}

\section{Counterproof arguments}

\section{All decimals are fractions, but not all fractions are decimals}

All fractions exist as the sum of one, in the form of $1 / n$. The question this paper poses is, how can the fractions $1 / 3+2 / 3$ exist as 1 , if $0.33(1 / 3)+0.66(2 / 3)=0.99 ?$

In decimal form, $0.33+0.66$ on their own do not equal to 1 , as there is a value of 0.1 that is missing. Instead, the accurate equation is $0.33+0.66+0.1=1$.

So, $A=1$ and $B=0.99$. Between the intervals of $A=1$ and $B=0.99,0.99(X) 1$ exists.

$0.9 \overline{9}>x<1$

$X$ is the value between 0.99 and 1 . This $X$ is a margin too narrow to contain. This margin was referenced in Fermat's Last Theorem, where he states that the margin is too narrow to contain. Although there have been differences in opinion and confusion on which "margin" he was referring to, this paper believes that the margin is the infinite number of possible values between 0.99 and 1.

This paper attempts to demonstrate that the sum of all fractions are not decimals.

In the example above, $A$ and $B$ are equalities. $A$ is the fraction form of $B$, with $B$ being the decimal form of $A$.

A as a fraction form is $[1 / 3+2 / 3=1]$

$B$ as a decimal form is $[0.33+0.66=0.99]$

However, if $A=1$ and $B=0.99$, then $A \neq B$

If $A \neq B$, this shows that the sum of all fractions are indeed not decimals.

As evident from the example above, not all fractions are decimals. If not all fractions are decimals, then not all fractions are non-integers. If not all fractions non-integers, then the sum of fractions are integers. The conclusion of this statement is that the sum of fractions can indeed be an integer, such as $1 / 3+2 / 3=1$.

\section{Zero is a Neutral Integer}

All integers are whole values, but not all integers are positive or negative. Zero exists as an integer, and it is neither positive nor negative. Therefore, zero can be considered as a neutral integer instead. Positive integers, negative integers, and neutral integers, such as zero, are all considered whole values. 
In order to delve into this concept further, an idea from Bowden will be used to demonstrate. [1] Elements of the Theory of Integers explains that a number is assigned to every group of objects, and a number belongs to every single object as well. The three groups are positive integers, neutral integers, and negative integers. The positive integers lie between the intervals of $(1, \infty)$ the neutral integer is

$(0)$, and the negative integers lie between the intervals of $(-1,(-\infty))$ The objects of the three groups of integers are the real numbers assigned to each domain. However, the neutral integer is a curious case where only one real number, zero, exists in its respective domain.

\section{The name and symbol of Zero}

The concept of a number is in itself a fundamental concept because a number is not defined in terms of anything simpler than itself. The concept of a number is formed inside our minds. An idea is associated with each number, which is then given a name. Thus, the names used for numbers are "one, two, six, etc." Numbers are also represented by symbols, such as " $1,2,6$, etc." Applying this logic to the neutral integer, 'zero' would be the name, and ' 0 ' would be the symbol associated with the idea of it. One can apply real-life logic to numbers quite easily, such as in the case of having two apples or three oranges. It is simple to count two apples on a counter, or three oranges at a farmers market. However, how does one logically apply "zero"? If zero is nothing, then 0 = nothing. So, the question is, how exactly nothing is counted? How does a thing exist as zero, or how does a thing exist at zero? The first law of thermodynamics, also known as the law of conservation of energy, states that energy is a constant in an isolated system, and it cannot be created nor destroyed, only transformed from one form to another. [2] Therefore, if the energy does not exist at zero, how does the value zero exist as nothing?

\section{4. "Nothing" can exist as "one"}

In this section, the following statement will be discussed and analyzed:

$0=$ nothing, and at the same time, $0=1$

If this is the case, then how can nothing exist as 1 ? According to the statement above, nothing can exist as one. Therefore, 0 can be 1 , but 1 cannot be 0 . Below are presented several tables that demonstrate by examples the validity of the statement.

Table 1. Wholes and Parts. The sum of a part is equal to a whole. This whole is then equal to one. If zero is considered a value, according to this table there are a total of 7 'whole/one values'. The 8 -whole/one values have 19 'parts of ones' in total.

\begin{tabular}{|l|l|l|l|l|l|}
\hline Ones/Wholes & $\begin{array}{l}\mathbf{0}^{\text {th }} \text { Part of the } \\
\text { ones }\end{array}$ & $\begin{array}{l}\mathbf{1}^{\text {st }} \text { part of the } \\
\text { ones }\end{array}$ & $\begin{array}{l}\mathbf{2}^{\text {nd }} \text { part of the } \\
\text { ones }\end{array}$ & $\begin{array}{l}\mathbf{3}^{\text {rd }} \text { part of the } \\
\text { ones }\end{array}$ & $\begin{array}{l}\mathbf{4}^{\text {th }} \text { part of the } \\
\text { ones }\end{array}$ \\
\hline 0$) 2 / 2=1$ & $1 / 2+1 / 2=2 / 2$ & & & & \\
\hline 1) $3 / 3=1$ & & $1 / 3+2 / 3=3 / 3$ & & & \\
\hline 2) $4 / 4=1$ & & $1 / 4+3 / 4=4 / 4$ & $2 / 4+2 / 4=4 / 4$ & & \\
\hline 3) $5 / 5=1$ & & $1 / 5+4 / 5=5 / 5$ & $2 / 5+3 / 5=5 / 5$ & & \\
\hline 4) $6 / 6=1$ & & $1 / 6+5 / 6=6 / 6$ & $2 / 6+4 / 6=6 / 6$ & $3 / 6+3 / 6=6 / 6$ & \\
\hline
\end{tabular}




\begin{tabular}{|l|l|l|l|l|l|} 
5) $7 / 7=1$ & & $1 / 7+6 / 7=7 / 7$ & $2 / 7+5 / 7=7 / 7$ & $3 / 7+4 / 7=7 / 7$ & \\
\hline 6$) 8 / 8=1$ & & $1 / 8+7 / 8=8 / 8$ & $2 / 8+6 / 8=8 / 8$ & $3 / 8+5 / 8=8 / 8$ & $4 / 8+4 / 8=8 / 8$ \\
\hline 7$) 9 / 9=1$ & & $1 / 9+8 / 9=9 / 9$ & $2 / 9+7 / 9=9 / 9$ & $3 / 9+6 / 9=9 / 9$ & $4 / 9+5 / 9=9 / 9$ \\
\hline
\end{tabular}

\section{Counterproof and counterexample to Beal's conjecture}

$A^{x}+B^{y}=C^{z}$

Where $A, B, C, x, y$, and $z$ are positive integers and $x, y, z>2$, then $A, B$, and $C$ must have a common prime factor.

Equivalently, there are no solutions to the above equation in positive integers $A, B, C, x, y, z$ with $A, B$ and $C$ being pairwise co-prime and all of $x, y$, and $z$ is greater than 2 .

$A^{x}+B^{y}=C^{z}$

Where $A, B, C, x, y$, and $z$ are neutral integers with $x, y$, and $z<2$, then $A, B$, and $C$ do not have a common prime factor.

Equivalently, there are solutions to the above equation in neutral integers $A, B, C, x, y, z$ with $A, B$ and $C$ not being pairwise co-prime and all of $x, y, z$ being less the 2 .

\section{Counterexample proof}

A demonstration to the counterexample is shown below:

$$
\begin{aligned}
& A=(1 / 2)^{(x)} \\
& B=(1 / 2)^{(y)} \\
& C=1.98650950590874287^{(z)} \\
& x=(1 / 128)=(0.0078125) \\
& y=(3 / 256)=(0.01171875) \\
& z=(5 / 256)=(0.001953)
\end{aligned}
$$

Apply to $A^{x}+B^{y}=C^{z}$

$(A)(1 / 2)^{\wedge} 0.0078125+(B)(1 / 2)^{\wedge} 0.01171875=\left(C^{1}\right) 1.98650950590874287$

\section{Part 2}

(C2) $1.98650950590874^{\wedge} 0.001953=1.00134139721571$

(Continue to raise $\mathrm{C} 2, \mathrm{C} 3, \mathrm{C} 4, \mathrm{C} 5$ and $\mathrm{C} 6$ to the power of $\mathrm{Z}$ )

$\left(C^{2}\right)=1.00134139721571$ 

$(C 3)=1.0000026179967$
$(C 4)=1.00000000511294$
$(C 5)=1.00000000000999$
$(C 6)=1.00000000000002$
$(C 7)=1$

Conclusion: Intervals $(C 7)=$, always equal 1

Table 2. Fractional Proportions of $A B C$ relative to one another (ABC) (Step 0 to Step 15). Step 0 indicates a constant value according to the proportional laws of the universe. Step 1 is concrete. The values of Step 8 are of note, as they coincide with the values used as proof for the counterexample of Beal's conjecture above.

\begin{tabular}{|c|c|c|c|c|c|c|}
\hline Steps & A & B & $\begin{array}{l}C^{0}(A+B)+C 1\left(C^{0}\right)^{2} \text { (multiply } \\
\text { the previous step by } 2 \text { as } \\
\text { well) }\end{array}$ & $X(-1 ?)$ & $\mathbf{Y}$ & $z(x+y)$ \\
\hline Step 0 & $1 / 2$ & $1 / 2$ & $1+1$ & $\begin{array}{l}1 / 128 \\
(0.0078125)\end{array}$ & $\begin{array}{l}3 / 256 \\
(0.01171875)\end{array}$ & $\begin{array}{l}5 / 256 \\
(0.01953125)\end{array}$ \\
\hline $\begin{array}{l}\text { Step } 1 \\
\text { (multiply step } \\
0 \text { by } 2 \text { ) }\end{array}$ & 1 & 1 & $2+2$ & 0.015625 & 0.0234375 & 0.0390625 \\
\hline $\begin{array}{l}\text { Step } 2 \\
\text { (multiply step } \\
1 \text { by } 2 \text { ) }\end{array}$ & 2 & 2 & $4+4$ & 0.03125 & 0.046875 & 0.078125 \\
\hline Step 3 & 4 & 4 & $8+16$ & $0.0625(?)$ & 0.09375 & 0.15625 \\
\hline Step 4 & 8 & 8 & $16+32$ & $0.0125(?)$ & 0.1874 & 0.3125 \\
\hline Step 5 & 16 & 16 & $32+64$ & 0.25 (?) & 0.375 & 0.625 \\
\hline Step 6 & 32 & 32 & $64+128$ & $0.5(?)$ & 0.75 & 1.25 \\
\hline Step 7 & 64 & 64 & $128+256$ & $1(?)$ & 1.5 & 2.5 \\
\hline Step 8 & 128 & 128 & $256+512$ & $2(1)$ & 3 & 5 \\
\hline Step 9 & 256 & 256 & $512+1024$ & $4(3)$ & 6 & 10 \\
\hline
\end{tabular}




\begin{tabular}{|l|l|l|l|l|l|l|} 
Step 10 & 512 & 512 & $1024+2048$ & $8(7)$ & 12 & 20 \\
\hline Step 11 & 1024 & 1024 & $2048+4096$ & $16(15)$ & 24 & 40 \\
\hline Step 12 & 2048 & 2048 & $4096+8192$ & $32(31)$ & 48 & 80 \\
\hline Step 13 & 4096 & 4096 & $8192+16,384$ & $64(63)$ & 96 & 160 \\
\hline Step 14 & 8192 & 8192 & $16,384+32,768$ & $128(127)$ & 192 & 320 \\
\hline Step 14 & 16,384 & 16,384 & $32,768+65,536$ & $256(255)$ & 384 & 640 \\
\hline Step 15 & 32,768 & 32,768 & $65,536+131072$ & $512(511)$ & 768 & 1280 \\
\hline
\end{tabular}

\section{Discussion}

Through the counterarguments above, the counterproof for Beal's conjecture can be demonstrated. This paper aims to prove that a special set of fractions exist as integers. The counterproof given in this paper explains that if a non-integer is a number written with a fractional component, then an integer is a number that is not written with a fractional component. For a thing to have a component, a whole value must already exist, for which the fraction behaves as a component. For a more thorough understanding, an example will be used to demonstrate this concept. For example, in " $41 / 2$ ", the " 4 " is the whole value, and the " $1 / 2$ " is the fractional component. Therefore, " $41 / 2$ " is a non-integer, because it contains both a whole value and a fractional component. If a fraction does not have a whole value, but rather two fractions, then it does not satisfy the requirements needed to be considered a non-integer. For a thing to be complete in itself, it must equal a whole number. Despite often not being seen as such, fractions are a thing equal in themselves. Fractions that are always equal to one are positive integers. Consequentially, it can be said that if a non-integer is a number written with a fractional component, then fractions can be considered integers. For example, in the case of $1 / 3+2 / 3=3 / 3$, since $1 / 3$ and $2 / 3$ are two parts that when added together, equal the sum of 1 , then $1 / 3+2 / 3$ are not to be considered non-integers. If $1 / 3+2 / 3$ are not non-integers, then $1 / 3+2 / 3$ are considered integers. Further examples and demonstration of this counterargument can be seen in Table 1, which shows that there are a total of 7 "whole/one values." This concept is also further shown in Table 2, where steps 1-15 are shown to demonstrate the counterproof for Beal's conjecture, through the values $A, B, C, x, y$, and $z$.

As mentioned earlier, "zero" is the only member of the group of neutral integers. The "ones" are the objects of the "zero." A specific set of parts is assigned to each "one." The group, the objects, and the assigned parts are equal to 1 in likeliness. However, they differ in their sameness. This is part of the reason why defining zero as an integer has proven to be difficult, and why the definition of it being a "neutral integer" is much more fitting. The parts are equal to 1 in the likeliness of sums. For example, 2/9+7/9=9/9, which is equal to a whole value of 1 when further divided. Therefore, $9 / 9$ exists as both a whole and a 1 . If it is possible to define " 0 ", then 1 is no longer the first number of a numerical sequence. If this is indeed the case, then " 0 " would be the first number of a numerical sequence.

On the other hand, parts split two ways. For example, $2 / 2$ is the only value in the "0 $0^{\text {th }}$ part of the ones" that splits two ways. In " $0^{\text {th }}$ part of the ones", $3 / 3$ splits into three equal $1 / 3$ parts, resulting in $1 / 3+1 / 3+1 / 3=3 / 3$. 
Therefore, it is not considered a $0^{\text {th }}$ part of the ones. All wholes/ones greater than $2 / 2$ are not considered to be " $0^{\text {th }}$ part of the ones", because they split more than two times.

The domain of the neutral integer seems to lie in the domain of $(0-(+1))$. In this case, the positive integers are values that lie in the domain of $(+1-(+))$. This would mean that the neutral integers are part of the positive integers. Therefore, a small fraction of positive integers is indeed neutral integers. These neutral integers appear to move on a linear path in a sum of parts until the number 1 is reached. Table 1 shows how 1 can be created when following any of the seven paths of the wholes. Once 1 is fully formed by the parts, it can be established as the first value in the domain of the positive integers, $(1-+$. The following values in the positive domain will then follow the numerical system, with numbers equaling the preceding numbers value plus 1 . This analysis and explanation show that parts can exist both as a composition and a division of things.

The counterexamples above show that there are in fact solutions to the Beal's conjecture equation in neutral integers $A, B, C, x, y, z$, with $A, B$, and $C$ not being pairwise coprime and all of $x, y, z$ being less than 2 , and that there are no solutions to the equation in positive integers $A, B, C, x, y, z$ with $A, B$, and $C$ being pairwise co-prime and all of $x, y$, and $z$ is greater than 2 .

\section{References}

1. Harold Edwards. Fermat's Last Theorem. A Genetic Introduction to Algebraic Number Theory. Graduate Texts in Mathematics, 50, 1997.

2. Davide Castelvecchi. Fermat's Last Theorem Earns Andrew Wiles the Abel Prize. Nature, 531 (2016), no. 7594, 287. DOI:10.1038/nature.2016.19552.

3. Science and Technology. The Guinness Book of World Records. Guinness Publishing Ltd. 1995.

4. Richard Daniel Mauldin. A Generalization of Fermat's Last Theorem: The Beal Conjecture and Prize Problem. Notices of the AMS. 44, (1997) no. 11, 1436-1439.

5. Joseph Bowden, Elements of the Theory of Integers, The MacMillan Company, London, 1903.

6. Sonntag, Richard Edwin, Claus Borgnakke, Gordon John Van Wylen, and Steve Van Wycik. Fundamentals of thermodynamics. Wiley, New York, 1998. 